\title{
Heavy Flavour Hadro-Production Cross-Sections
}

\author{
Hermine K. Wöhri and Carlos Lourenço
}

CERN/EP, Geneva, Switzerland

\begin{abstract}
Hadro-production data on charm and beauty absolute cross-sections, collected by experiments at CERN, DESY and Fermilab, are reviewed. The measurements, corrected for the time evolution' of the branching ratios, are compared to calculations done with Pythia, as a function of the collision energy, using the latest parametrizations of the parton densities. We then estimate some charm and beauty production cross-sections relevant for future measurements, including nuclear effectes in the PDFs. We finish by briefly addressing the relevance, in heavy-ion collisions, of beauty production as feed-down for $\mathrm{J} / \psi$ production.
\end{abstract}

In the near future, several heavy-ion experiments (at the SPS, RHIC and LHC) will be sensitive to the production of open charm and open beauty production. It is, therefore, timely to review what is known today about production yields and estimate what can be expected for these future measurements.

The data used in this study were obtained with proton and pion beams at energies from $E_{\text {lab }}=200$ to $920 \mathrm{GeV}$ and from $\sqrt{s}=0.63$ to $1.96 \mathrm{TeV}$, being summarized in Table 1 . The reported beauty production values refer to a global mixture of beauty hadrons, mostly measured by looking at high $p_{\mathrm{T}}$ single muons, dimuons or even trimuons, except for $\mathrm{CDF}$ which gives $\mathrm{B}^{+}$cross-sections. While most experiments had full coverage in the positive $x_{\mathrm{F}}$ region, the E789 experiment covered a rather small window. All experiments using nuclear targets assumed a linear dependence of heavy flavour production on the mass number of the target nucleus, to derive the cross-section per nucleon. The different measurements on charm production cross sections were normalized to common branching ratios, using the PDG 2002 tables [1], to remove the 'time evolution' of these values. We have also updated the systematic errors of the published values, to reflect the smaller uncertainties of the most recent branching ratios. If the $\mathrm{D}$ mesons were searched in more than one decay channel, the performed corrections were weighted according to the number of observed events in each of the channels. While the data on D meson production collected with pion beams were published for the positive $x_{\mathrm{F}}$ range, data obtained with proton beams were mostly published for the full $x_{\mathrm{F}}$ range. To ease their comparison, all measurements were normalized to the positive $x_{\mathrm{F}}$ range. The very recent CDF charm measurement was done for $p_{\mathrm{T}}>5.5 \mathrm{GeV} / \mathrm{c}$.

After having collected the available charm and beauty cross-sections we can compare their $\sqrt{s}$ dependence with calculations performed with Pythia [26], a Monte-Carlo event generator used by many experiments. The calculations used version 6.208 , with its default settings. In particular, the $c$ and $b$ quark masses are 1.5 and $4.8 \mathrm{GeV} / \mathrm{c}^{2}$, respectively. The neutral and charged $D$ meson production cross-sections in $\pi^{-} p$ and pp collisions are compared in Fig. 1 with the Pythia curves obtained with different PDFs [27]. After normalizing the curves to the data (not including the CDF point), fitting 
an empirical K-factor, none of the curves can be excluded.

While in pion induced collisions the $\mathrm{D}^{+} / \mathrm{D}^{-}$and $\mathrm{D}^{0} / \overline{\mathrm{D}^{0}}$ data require similar $\mathrm{K}$-factors, of around 1.5 , in $\mathrm{p}-\mathrm{A}$ collisions the calculated charged $\mathrm{D}$ meson cross-sections are much lower than the measured values. Figure 2 (left) shows the charged to neutral D meson ratio measured in proton induced reactions. Their average is two times higher than the value given by Pythia, 0.32, and expected assuming equal production probabilities of the $\mathrm{D}$ and $\mathrm{D}^{*}$ states and using known $\mathrm{D}^{*}$ branching ratios. It is worth noting that the data collected with pion-induced collisions do not show such a strong discrepancy.

TABLE 1. Charm and beauty measurements used in this study.

\begin{tabular}{|c|c|c|c|c|c|c|}
\hline & \multicolumn{2}{|c|}{ Exp. / Publication } & \multirow{2}{*}{$\frac{\text { Target(s) }}{p}$} & \multirow{2}{*}{$\frac{D^{0}+\overline{D^{0}} \text { events }}{5}$} & \multirow{2}{*}{$\frac{\mathrm{D}^{+}+\mathrm{D}^{-} \text {events }}{10}$} & \multirow{2}{*}{$\frac{\text { acc. window }}{x_{\mathrm{F}}>-0.1}$} \\
\hline \multirow{6}{*}{ 志 } & NA16 & [2] 1984 & & & & \\
\hline & NA27 & [3] 1988 & $\mathrm{p}$ & 98 & 119 & $x_{\mathrm{F}}>-0.1$ \\
\hline & E743 & [4] 1988 & $\mathrm{p}$ & 10 & 46 & $x_{\mathrm{F}}>-0.1$ \\
\hline & E653 & [5] 1991 & emulsion & 108 & 18 & $x_{\mathrm{F}}>-0.2$ \\
\hline & E789 & [6] 1994 & $\mathrm{Be}, \mathrm{Au}$ & $>4000$ & - & $0.00<x_{\mathrm{F}}<0.08$ \\
\hline & E769 & [7] 1996 & $\mathrm{Be}, \mathrm{Al}, \mathrm{Cu}, \mathrm{W}$ & 136 & 159 & $x_{\mathrm{F}}>-0.1$ \\
\hline 次 & $\mathrm{CDF}$ & [8] 2002 & $\mathrm{p} \overline{\mathrm{p}}$ & 36804 & 28361 & $p_{\mathrm{T}}>5.5 \mathrm{GeV} / \mathrm{c}$ \\
\hline \multirow{9}{*}{ 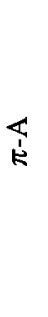 } & NA16 & [2] 1984 & $\mathrm{p}$ & 4 & 9 & $x_{\mathrm{F}}>-0.1$ \\
\hline & NA27 & [9] 1986 & $\mathbf{p}$ & 49 & 14 & $x_{\mathrm{F}}>0.0$ \\
\hline & NA32 & [10] 1988 & $\mathrm{Si}$ & 75 & 39 & $x_{\mathrm{F}}>0.0$ \\
\hline & NA32 & [11] 1991 & $\mathrm{Cu}$ & 543 & 249 & $x_{\mathrm{F}}>0.05$ \\
\hline & E653 & [12] 1992 & emulsion & 328 & 351 & $\dot{x}_{\mathrm{F}}>0.0$ \\
\hline & E769 & [7] 1996 & $\mathrm{Be}, \mathrm{Al}, \mathrm{Cu}, \mathrm{W}$ & $62 / 353$ & $73 / 414$ & $x_{\mathrm{F}}>-0.1$ \\
\hline & WA92 & [13] 1997 & $\mathrm{Cu}, \mathrm{W}$ & 3873 & 3299 & $x_{\mathrm{F}}>0.0$ \\
\hline & E791 & [14] 1999 & $\mathrm{C}, \mathrm{Pt}$ & 88990 & - & $x_{F}>-0.1$ \\
\hline & E706 & [15] 1997 & $\mathrm{Be}, \mathrm{Cu}$ & 一 & 110 & $x_{\mathrm{F}}>-0.2$ \\
\hline
\end{tabular}

\begin{tabular}{|c|c|c|c|c|c|}
\hline & \multicolumn{2}{|c|}{ Exp. / Publication } & Target(s) & $B \bar{B}$ events & acceptance window \\
\hline $\begin{array}{l}\mathbb{1} \\
\dot{R}\end{array}$ & $\begin{array}{l}\text { NA10 } \\
\text { WA78 } \\
\text { E653 } \\
\text { E672/E706 } \\
\text { WA92 } \\
\end{array}$ & $\begin{array}{l}{[16] 1988} \\
{[17] 1989} \\
{[18] 1993} \\
{[19] 1995} \\
{[20] 1998} \\
\end{array}$ & $\begin{array}{c}\mathrm{W} \\
\mathrm{U} \\
\text { emulsion } \\
\mathrm{Be} \\
\mathrm{Cu} \\
\end{array}$ & $\begin{array}{c}43 \\
12 \\
9 \pm 3 \\
8 \pm 3.3 \\
26 \\
\end{array}$ & $\begin{array}{c}x_{\mathrm{F}}>0 \\
x_{\mathrm{F}}>0 \\
x_{\mathrm{F}}>-0.3 \\
x_{\mathrm{F}}>0 \\
-0.5<x_{\mathrm{F}}<0.6\end{array}$ \\
\hline 岩 & $\begin{array}{l}\text { E789 } \\
\text { E771 } \\
\text { HERA-B }\end{array}$ & $\begin{array}{l}{[21] 1995} \\
{[22] 1999} \\
{[23] 2002}\end{array}$ & $\begin{array}{c}\mathrm{Au} \\
\mathrm{Si} \\
\mathrm{C}, \mathrm{Ti}\end{array}$ & $\begin{array}{c}19 \pm 5 \\
15 \\
10.5_{-9.2}^{+13.1}\end{array}$ & $\begin{array}{c}0<x_{\mathrm{F}}^{\mathrm{J} / \Psi}<0.1, p_{\mathrm{T}}^{\mathrm{J} / \Psi}<2 \mathrm{GeV} / \mathrm{c} \\
x_{\mathrm{F}}>-0.25 \\
-0.25<x_{\mathrm{F}}^{\mathrm{J} / \Psi}<0.15\end{array}$ \\
\hline '告 & $\begin{array}{l}\text { UA1 } \\
\text { CDF }\end{array}$ & $\begin{array}{l}{[24] 1991} \\
{[25] 2002} \\
\end{array}$ & $\begin{array}{l}\mathrm{p} \overline{\mathrm{p}} \\
\mathrm{p} \overline{\mathrm{p}}\end{array}$ & $\begin{array}{c}2859 \\
387 \pm 32 \\
\end{array}$ & $\begin{array}{l}|y|<1.5, p_{\mathrm{T}}^{\mu}>6 \mathrm{GeV} / \mathrm{c} \\
|y|<1.0, p_{\mathrm{T}}^{\mu}>6 \mathrm{GeV} / \mathrm{c}\end{array}$ \\
\hline
\end{tabular}

Figure 3 shows the energy dependence of the total $c \bar{c}$ production cross-section, derived by adding the measured neutral and charged $\mathrm{D}$ meson values, and considering that the production of other charmed hadrons $\left(\Lambda_{c}, \mathrm{D}_{s}\right.$, etc) is $20 \%$ of the total yield. Before being included in this figure, the CDF value was extrapolated down to zero $p_{\mathrm{T}}$, using the distributions given by Pythia with the $\left\langle k_{\mathrm{T}}^{2}\right\rangle$ parameter varied between 1 and $2(\mathrm{GeV} / \mathrm{c})^{2}$. An error of $40 \%$ was added to account for the uncertainty in this extrapolation. Once Pythia is normalized to the available data we can estimate the charm production cross-sections 

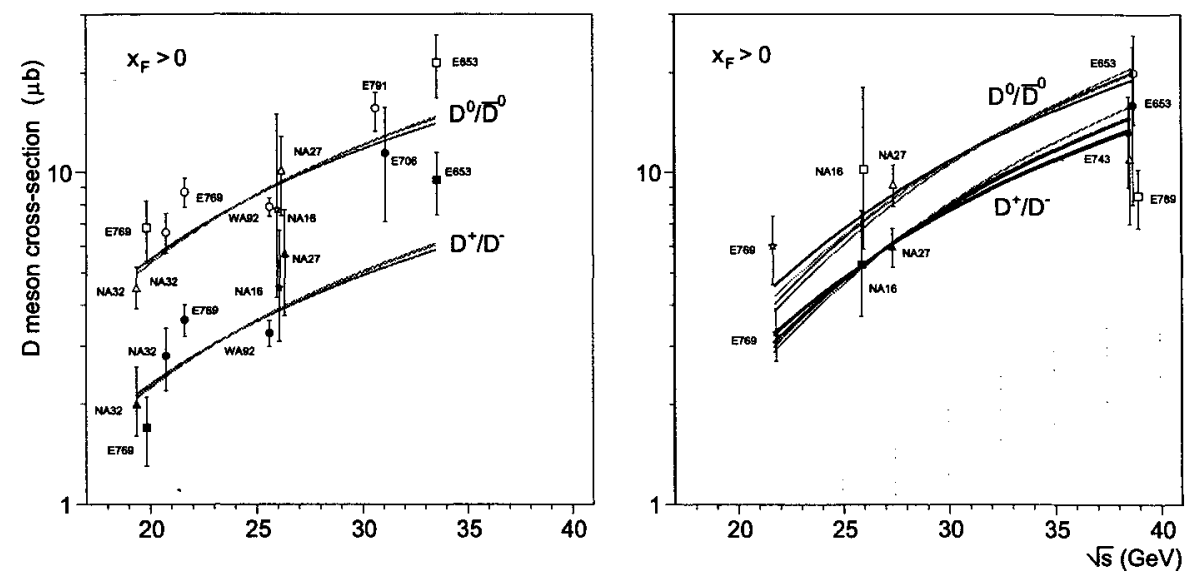

FIGURE 1. Energy dependence of the neutral (open symbols) and charged (closed symbols) D meson production cross-sections in $\pi^{-} \mathrm{p}$ (left) and pp (right) collisions.
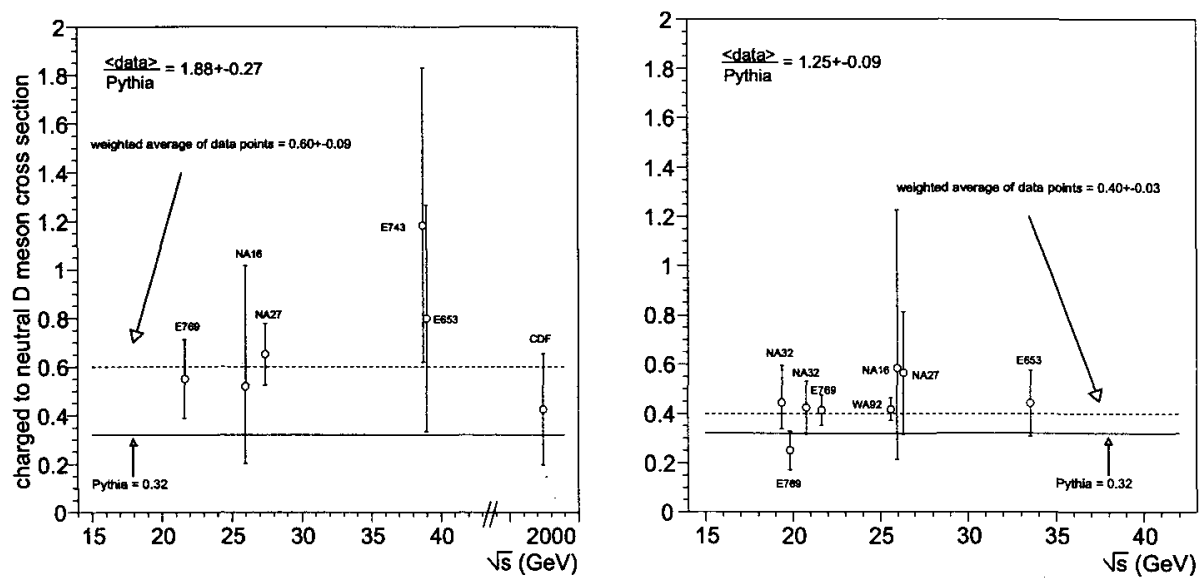

FIGURE 2. Ratio of charged to neutral D meson yields in proton (left) and pion (right) induced collisions.

for any other energies. The five curves that go below the CDF data point represent $\mathrm{LO}$ calculations (pure gluon fusion and $q \bar{q}$ annihilation) while the four higher curves include initial and final state parton showers, gluon splitting and flavour excitation, also simulated within Pythia.

For RHIC energies, $\sqrt{s}=200$ and $500 \mathrm{GeV}$, the curves indicate $c \bar{c}$ cross-sections (per nucleon-nucleon collision) clustering around $\sim 1$ and $\sim 3 \mathrm{mb}$, respectively, but with uncertainties around $100 \%$. For the NA60 experiment [28], the spread in the curves is much smaller, giving $\sim 5 \mu \mathrm{b}$ and $20 \mu \mathrm{b}$, respectively for In-In collisions at $E_{\text {lab }}=158 \mathrm{GeV}$ and p-A collisions at $400 \mathrm{GeV}$. Anti-shadowing of the PDFs was included using the EKS 98 model [29]. 


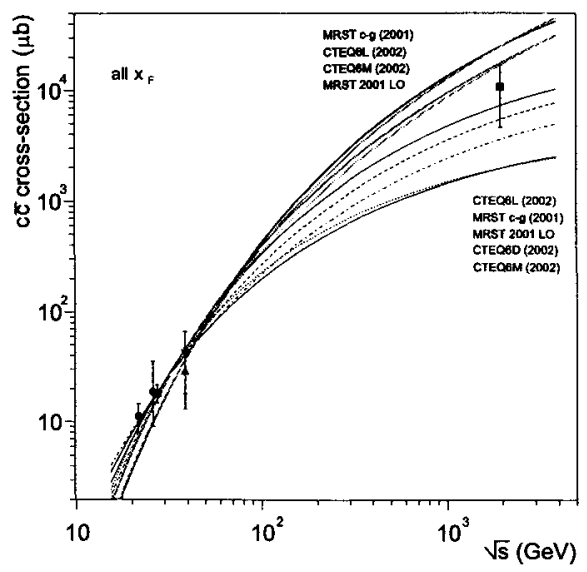

FIGURE 3. $c \bar{c}$ production cross-sections versus collision energy.
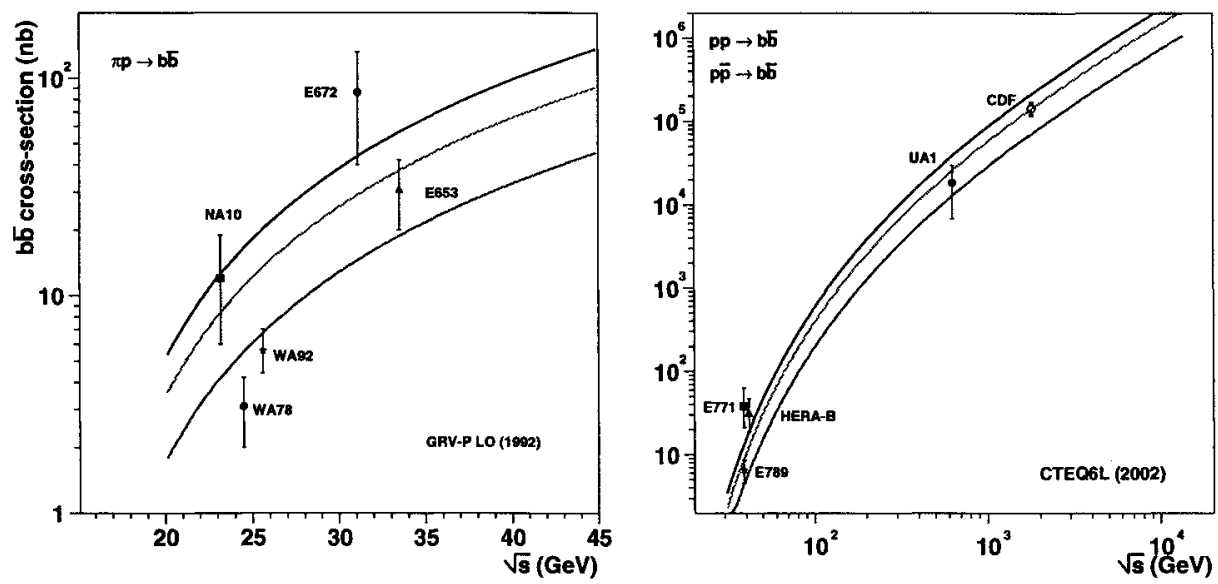

FIGURE 4. $b \bar{b}$ production cross-sections in pion (left) and proton (right) induced collisions.

Figure 4 shows the few available measurements of beauty production cross-sections in pion (left) and proton (right) induced collisions. Unfortunately, the data points are considerably spread around, with factors of 5 between measurements made at essentially the same energy (NA10 and WA78; E771 and E789). To minimize the number of standard deviations between the data points and the normalized curves, the pion induced collisions prefer $\mathrm{K}$-factors below 1.4 while the proton data can accomodate values between 1 and 3 . Therefore, rough estimates for the $b \vec{b}$ production cross-sections, per nucleon-nucleon collision, for the heavy-ion collider experiments are $\sim 2 \mu \mathrm{b}$ at RHIC and $\sim 350 \mathrm{mb}$ at the LHC. These values include nuclear effects in the PDFs, assuming $\mathrm{Pb}$ colliding nuclei. Clearly, better data is needed in the beauty sector, including a measurement of the nuclear effects in proton-nucleus collisions. 
At collider energies, beauty production is a very important source of $\mathrm{J} / \psi$ mesons. CDF observed that beauty decays represent more than $15 \%$ of the total $\mathrm{J} / \psi$ yield [30], at $\sqrt{s}=1.8 \mathrm{TeV}$, and for $\mathrm{J} / \psi$ mesons of $p_{\mathrm{T}}$ above $5 \mathrm{GeV} / c$. Since beauty production is expected to scale linearly with the mass number of the colliding nuclei, while $\mathrm{J} / \psi$ production scales as $A^{0.92}$, due to the normal nuclear absorption, in $\mathrm{Au}-\mathrm{Au}$ collisions the relative fraction of $\mathrm{J} / \psi$ mesons resulting from beauty decays should be 2.3 times higher. If direct $\mathrm{J} / \psi$ production is further suppressed in heavy-ion collisions (NA50 measured a factor 2 in central $\mathrm{Pb}-\mathrm{Pb}$ collisions at the SPS), beauty production might account for more than $50 \%$ of the observed $\mathrm{J} / \psi$ yield, maybe already at RHIC energies. This observation underlines the importance of upgrading the RHIC experiments with vertexing detectors, in view of a proper interpretation of the measured $J / \psi$ suppression pattern.

We thank A. David, A. Devismes and A. Morsch for considerable help in part of this work. We also thank M. Mangano, F. Mokler, L. Ramello, C. Salgado, J. Schukraft and P. Weilhammer for very useful discussions.

\section{REFERENCES}

1. K. Hagiwara et al. (PDG), Phys. Rev. D66 (2002) 010001.

2. M. Aguilar-Benitez et al. (NA16 Coll.), Phys. Lett. B135 (1984) 237.

3. M. Aguilar-Benitez et al. (NA27 Coll.), Z. Phys. C40 (1988) 321.

4. R. Ammar et al. (E743 Coll.), Phys. Rev. Lett. 61 (1988) 2185.

5. K. Kodoma et al. (E653 Coll.), Phys. Lett. B263 (1991) 573.

6. M.J. Leitch et al. (E789 Coll.), Phys. Rev. Lett. 72 (1994) 2542.

7. G.A. Alves et al. (E769 Coll.), Phys. Rev. Lett. 77 (1996) 2388.

8. D. Acosta et al. (CDF Coll.), hep-ex/0307080.

9. M. Aguilar-Benitez et al. (NA27 Coll.), Z. Phys. C31 (1986) 491.

10. S. Barlag et al. (NA32 Coll.), Z. Phys. C39 (1988) 451.

11. S. Barlag et al. (NA32 Coll.), Z. Phys. C49 (1991) 555.

12. K. Kodoma et al. (E653 Coll.), Phys. Lett. B284 (1992) 461.

13. M. Adamovich et al. (WA92 Coll.), Nucl. Phys. B495 (1997) 3.

14. E.M. Aitala et al. (E791 Coll.), Phys. Lett. B462 (1999) 225.

15. L. Apanasevich et al. (E706 Coll.), Phys. Rev. D56 (1997) 1391.

16. P. Bordalo et al. (NA10 Coll.), Z. Phys. C39 (1988) 7.

17. M.G. Catanesi et al. (WA78 Coll.), Phys. Lett. B 231 (1989) 328.

18. K. Kodoma et al. (E653 Coll.), Phys. Lett. B 303 (1993) 359.

19. R. Jesik et al. (E672 and E706 Coll.), Phys. Rev. Lett. 74 (1995) 495.

20. M. Adamovich et al. (WA92 Coll.) Nucl. Phys. B519 (1998) 19.

21. D.M. Jansen et al. (E789 Coll.), Phys. Rev. Lett. 74 (1995) 3118.

22. T. Alexopoulos et al. (E771 Coll.), Phys. Rev. Lett. 82 (1999) 41.

23. I. Abt et al. (HERA-B Coll.), Eur. J. Phys. C26 (2002) 345.

24. C. Albajar et al. (UA1 Coll.), Phys. Lett. B256 (1991) 121.

25. D. Acosta et al. (CDF Coll.), Phys. Rev. D65 (2002) 052005.

26. T. Sjöstrand et al., Comp. Phys. Comm. 135 (2001) 238.

27. H. Plothow-Besch, Comp. Phys. Comm. 75 (1993) 396; J. Pumplin et al., JHEP 0207 (2002) 012; A.D. Martin et al., Phys. Lett. B531 (2002) 216; Eur. Phys. J. C23 (2002) 73.

28. NA60 Proposal, CERN/SPSC 2000-010; http://cern.ch/na60

29. K.J. Eskola et al., Eur. Phys. J. C9 (1999) 61.

30. F. Abe et al. (CDF Coll.), Phys. Rev. Lett. 79 (1997) 572. 